$>1 \mathrm{~cm}(\mathrm{p}=0.008)$, diffuse small bowel adhesion/thickening $(\mathrm{p}=0.018)$ and lesser sac lesion $>1 \mathrm{~cm}(\mathrm{p}<0.001)$ were classified as predicting factors for suboptimal surgery. However, only CT features of retroperitoneal lymph nodes above renal hilum $>1 \mathrm{~cm}(\mathrm{p}=0.046)$ and lesser sac lesion $>1 \mathrm{~cm}(\mathrm{p}=0.004)$ were significant predicting factors for suboptimal surgery in multivariate analysis.

Conclusion: The preoperative predicting factors of suboptimal primary cytoreductive surgery in advanced stage ovarian cancer were retroperitoneal lymph nodes above renal hilum $>1 \mathrm{~cm}$ and lesser sac lesion $>1 \mathrm{~cm}$ on CT scan. The suboptimal cytoreduction rate was $62.5 \%$.

Poster (027)

Epithelial Ovarian Cancer including Borderline Tumor https://doi.org/10.3802/jgo.2021.32.S1.027

\section{Wip1 suggested as an independent predictor for poor overall survival through chemoresistance in specifically advanced- stage ovarian clear cell carcinomas}

\section{Chenyang Xu, Takeo Minaguchi, ' Nan Qi, Hiroya Itagaki, Ayumi Shikama, Nobutaka Tasaka, Azusa Akiyama, Sari Nakao, Hiroyuki Ochi, Toyomi Satoh \\ University of Tsukuba, Tsukuba, Japan (minaguchit@md.tsukuba.ac.jp)}

Objective: Although ovarian clear cell carcinoma (OCCC) tends to be diagnosed at an early stage, advanced-stage disease shows poor prognosis due to chemoresistance unlike the more common high-grade serous carcinoma.

Methods: We explored the differential roles of the Wip1-p38p53 DNA damage response pathway in early or advanced-stage OCCCs, respectively. We performed immunohistochemistry of Wip1, nuclear/cytoplasmic phospho-p38, p53 and phospho-p53 in OCCCs from consecutive 143 patients. Clinicopathological and prognostic data were retrospectively reviewed and correlated with the protein expressions.

Results: High Wip1 expression was significantly associated with positive $\mathrm{p} 53$, which was significantly associated with low nuclear phospho-p38 expression ( $\mathrm{p}=0.011$ and 0.0094 , respectively). In the early-stage diseases $(n=102)$, patients with positive $\mathrm{p} 53$ showed trends toward worse overall survival (OS) ( $p=0.062)$. Whereas in the advanced-stage diseases $(n=41)$, patients with highWip1 expression showed significantly worse OS ( $\mathrm{p}=0.0012$ ). The univariate and multivariate analyses for prognostic factors in the advanced-stage diseases indicated that high Wip1 expression was significant and independent for worse OS $(\mathrm{p}=0.011)$, but not in the early-stage diseases. Furthermore, high Wip1 showed a trend toward shorter treatment-free interval in advanced stages, but not in early stages ( $\mathrm{p}=0.083$ vs. $\mathrm{p}=0.93$ ). Conclusion: Wip1 appears to play a significant role for the prognosis of OCCCs through chemoresistance specifically in advanced stages, suggesting that Wip1 may serve as a reasonable therapeutic target for improving the poor prognosis of advanced-stage OCCC.

Poster (028)

Epithelial Ovarian Cancer including Borderline Tumor https://doi.org/10.3802/jgo.2021.32.S1.028

\section{Ambulatory chemotherapy in epithelial ovarian cancer patients}

\section{Suwanit Therasakvichya, ${ }^{*}$ Siree Limtiamchareon}

Faculty of Medicine, Siriraj Hospital, Mahidol University, Salaya, Thailand (suwanit.the@mahidol.ac.th)

Objective: Chemotherapy is a very effective for treatment of epithelial ovarian cancer (EOC) in not only primary but also in recurrence disease. According to health care policy to date, standard chemotherapy (paclitaxel plus carboplatin) should be managed as outpatient care. To determine the response rate and the toxicity of a 3-hour paclitaxel plus carboplatin administered as ambulatory chemotherapy for primary or recurrence EOC.

Methods: Retrospective review of medical records between April 2010 and July 2012. Seventy-nine EOC patients with mean age of 55 years old who were treated with adjuvant or salvage chemotherapy (intravenous paclitaxel $175 \mathrm{mg} / \mathrm{m}^{2}$ drip in 3 hours plus carboplatin area under the curve [AUC] 5 drip in 1 hour) every 21 days for 6 cycles.

Results: Twenty-four (36.4\%) patients were in early stage (I-II) and $42(63.6 \%)$ patients were in advanced stage (III-IV). Of $83.5 \%$ of the patients were in primary treatment and $16.5 \%$ were in recurrence. The complete response rates were $79.2 \%$, $64.3 \%$, and $46.2 \%$ in early, advanced, and recurrence cases, respectively. Four patients had hypersensitivity reaction during the first administration of paclitaxel, another patient had hypersensitivity reaction after carboplatin infusion. Only 3.8\% (3/79) developed febrile neutropenia which were manageable. Fourteen patients $(17.7 \%)$ had grade III-IV neutropenia without any symptoms. Most of the patients complained of grade I neuropathy.

Conclusion: Three-hour $175 \mathrm{mg} / \mathrm{m}^{2}$ paclitaxel plus 1 hour carboplatin AUC 5 is effective and quite safe for ambulatory care. However, hypersensitivity reaction should be accounted for especially during the first course of administration. 